\title{
Contents - Inhalt, Vol. 20, No. 5, 1997
}

\section{Review Articles}

362 Neuronavigation - the Gentle Way of Removing Brain Tumours

Sobottka, S.B.; Steinmetz, A.; Schackert, G. (Dresden) 372 Haematopoietic Myeloid Growth

Factors in the

Treatment of Acute Myelogenous Leukaemia: Players on or offStage?

Fey, M.F.; Tobler, A. (Bern) 380 Topotecan (Hycamtin $\left.{ }^{\circledR}\right)$ : Potent Cytostatic Action by Selective Topoisomerase I Inhibition

von Pawel, J. (München) 387 Clinical Relevance of Detection of Tumor Cells

in the Bone Marrow of Primary Breast Cancer Patients:

A Review

Harbeck, N. (München); Abdulsalam, A. (Damascus); Jänicke, F. (Hamburg) 393

Methodological Problems in Evaluating Duration of Response

to Therapy in Cancer Clinical Trials

Schroder, T.; Schumacher, M. (Freiburg i.Br.)

Short Review

Übersichtsarbeiten

362 Neuronavigation - der schonende Weg in der Entfernung von Hirntumoren

Sobottka, S.B.; Steinmetz, A.; Schackert, G (Dresden) 372 Hämatopoietische myeloische

Wachstumsfaktoren in der

Therapie akuter myeloischer Leukämien: Eine Hoffnung

zu Grabe getragen?

Fey, M.F.; Tobler, A. (Bern) 380 Topotecan (Hycamtin $\left.{ }^{\circledR}\right)$ : Hochwirksame zytostatische

Aktivität bei selektiver Topoisomerase-I-Inhibition

von Pawel, J. (München) 387 Klinische Bedeutung des Nachweises von Tumorzellen

im Knochenmark beim primären Mammakarzinom:

Eine Übersicht

Harbeck, N. (München); Abdulsalam, A. (Damascus); Jänicke, F. (Hamburg) 393

Methodologische Probleme zur Bestimmung der

Ansprechdauer auf die Therapie in klinischen Krebsstudien

Schroder, T.; Schumacher, M. (Freiburg i.Br.)

Kurze Übersicht

400 Current Opinion for Diagnosis and Treatment of Merkel Cell Carcinoma

Hartschuh, W. (Heidelberg)

Original Articles

406 Bendamustine, Methotrexate, Mitoxantrone, and Prednisolone (BMMP) for the Treatment of Relapsed or Refractory High-Grade Non-Hodgkin's Lymphoma 
Kahl, C. (Magdeburg); Herold, M. (Erfurt); Höffkes, H.-G; Franke, A. (Magdeburg) 410

Expression of Epidermal Growth Factor Receptor in Lymph Node Metastases of Head and Neck Squamous Cell Carcinomas

Riedel, F.; Bergler, W.; Baker-Schreyer, A.; Juncker, C; Hörmann, K. (Mannheim)

Commentary

416 Do We Still Need Estrogen and Progesterone Receptor Measurements for Breast Cancer?

Lüftner, D.; Possinger, K. (Berlin)

Oncological Education

418 Prevention and Treatment of Radiation-Induced Bowel Inj uries

Schratter-Sehn, A.U. (Wien)

400 Diagnose und Therapie des Merkelzellkarzinoms

Hartschuh, W. (Heidelberg)

Originalarbeiten

406 Bendamustin, Methotrexat, Mitoxantron und Prednisolon (BMMP) in der Behandlung rezidivierter oder refraktärer hochmalignerNon-Hodgkin-Lymphome

Kahl, C. (Magdeburg); Herold, M. (Erfurt); Höffkes, H.-G; Franke, A. (Magdeburg) 410 Die

Expression des EGF-Rezeptors in

Lymphknotenmetastasen von Plattenepithelkarzinomen aus

dem Kopf-Hals-Bereich

Riedel, E; Bergler, W; Baker-Schreyer, A.; Juncker, C; Hörmann, K.

(Mannheim)

Kommentar

416 Benötigen wir noch Östrogen- und Progesteronrezeptor-bestimmungen für das

Mammakarzinom?

Lüftner, D.; Possinger, K. (Berlin)

Onkologische Fortbildung

418 Pravention und Behandlung radiogener Darmkomplikationen

Schratter-Sehn, A.U. (Wien)

Continued on page 360

Fortsetzung aufSeite 360

Bibliographischer Hinweis: Inhaltsverzeichnisse dieser Zeitschrift erscheinen regelmäßig in current contents ${ }^{\circledR}$ sowie in anderen bibliographischen Diensten.

Contents - Inhalt

ONKOLOGIE

Vol. 20, Issue 5, October 1997

Vol. 20, Heft 5, Oktober 1997

Clinical Information

422 Oncological Clinical Research in Germany - Pharmaceutical Industry's Viewpoint

Hilgard, P.; Sindermann, H. (Frankfurt/M.) 426 Breast Cancer Prognostic and Therapy-Relevant

Factors: Results of a Consensus Conference

Graeff, H.; Wilmanns, W.; Jänicke, R; Sauer, H.; Classen, S. (München)

Klinische Informationen

422 Klinisch-onkologische Forschung in Deutschland aus Sicht der pharmazeutischen Industrie 
Hilgard, P.; Sindermann, H. (Frankfurt/M.) 426 Prognostische und therapierelevante Faktoren beim

Mammakarzinom - Ergebnisse einer Konsensuskonferenz

Graeff, H; Wilmanns, W.; Jänicke, E; Sauer, H.; Classen, S. (München)

Congress Report

Kongreßbericht

430 Spontaneous Remissions in Cancer Disease.

International Symposion, April 17-19,1997, Heidelberg

Zürner, P.; Heim, M.E. (Bad Sooden-Allendorf)

430 Spontanremissionen bei Krebserkrankungen.

Internationales Symposion 17. bis 19.4.1997, Heidelberg

Zürner, P.; Heim, M.E. (Bad Sooden-Allendorf)

433 Book Reviews

433 Buchbesprechungen

435 Industrial Forum

435 Industrieforum

357 Imprint

436 Instructions to Authors

357 Impressum

U3 Hinweise für Autoren (3. umschiagseíte)

Contents of Next Issues

Review Article

Hormone Replacement Therapy and Gynecologic Cancer

Krauß, K.; Rossmanith, W.G. (Ulm)

\section{Short Review}

The Use of Serological Tumor Markers for Malignant Melanoma

Hauschild, A. (Kiel)

Original Article

Interferon- $\alpha$ and Interferon- $\gamma$ in Advanced Renal Cell Carcinoma: Treatment Results, Survival, and Prognostic Factors in 81 Patients

Lümmen, G.; Sperling, H.; Luboldt, H.; Otto, T; Rübben, H.

(Essen)

Oncological Education Hormonersatztherapie nach Mammakarzinom

Kubista, E. (Wien)

Clinical Information

Zum Risiko von Zweitmalignomen anderer Lokalisation nach Diagnose einer Krebserkrankung der weiblichen Brustdrüse. Daten des bevölkerungsbezogenen Krebsregisters Saarland (19701992)

Bertz, I; Görsch, B. (Berlin); Stegmaier, C; Ziegler, H. (Saarbrücken) 
Praxis und Probleme bei der Entsorgung von Zytostatikaabfällen Scherrer, M.; Daschner, F. (Freiburg i. Br.)

360 